\title{
AN EXACT ANALYSIS OF THE LMS ALGORITHM WITH TONAL REFERENCE SIGNALS IN THE PRESENCE OF FREQUENCY MISMATCH
}

\author{
Hideaki Sakai and Yoichi Hinamoto \\ Dept. of Systems Science, Graduate School of Informatics, Kyoto University, \\ Kyoto 606-8501, Japan \\ phone: +81-75-753-5492, fax: +81-75-753-4755 \\ email: hsakai@i.kyoto-u.ac.jp
}

\begin{abstract}
An exact analysis is presented for the LMS algorithm with tonal reference signals in the presence of frequency mismatch. First, the time-varying linear system describing the LMS algorithm is converted into a time-invariant linear system. Then, a necessary and sufficient condition about the step sizes for convergence of the algorithm is derived using the Lyapunov function method and a transient behavior is analyzed. Finally, the effects of observation noise and frequency mismatch are examined without any approximations. The validity of the obtained results is shown by simulations.
\end{abstract}

\section{INTRODUCTION}

The LMS algorithm is most frequently used in the field of adaptive filtering because of its simplicity in implementation and robustness of performance. For the convergence analysis, the independence assumption (IA) is often made for stochastic situations [1]. But this assumption does not hold for most of the adaptive filtering problems. The results based on IA are sometimes justified by the averaging method [2]. But the averaging method relies on the slow adaptation limit so that the results are valid for small values of the step size parameter.

In general deterministic situations an exact analysis using the Lyapunov function method is presented in [2]. Also, more general results using passivity relations are presented in [3]. For more specific situation, a case where a pair of a sinusoid and a cosinusiod is used as reference signals is treated in [1] where the z-transform method is used. Recently a new method is proposed in [4] based on the eigen decomposition of the correlation matrix of the two-dimensional input vector whose elements are complex sinusoids.

In this paper, we treat the general case of $p$-dimensional input vector whose elements are complex sinusoids. First we convert the time-varying linear system describing the LMS algorithm into a time-invariant linear system. Then, a necessary and sufficient condition about the step size parameters for convergence of the algorithm is derived using the Lyapunov function method. The transient behavior is analyzed and the optimal selection of the step size parameters for minimizing the transient effect is discussed. Also, the effect of observation noise is considered and an exact expression of the excess mean square error (EMSE) is derived. In the adaptive filtering literature the expression for EMSE has been derived under some approximating assumption. Finally, the effect of frequency mismatch is examined. In [5] an analysis of this effect is made based on the averaging method. Here an exact result is presented in this case, too. The validity of the obtained results is shown by simulations.

\section{THE LMS ALGORITHM WITH TONAL REFERENCE SIGNALS AND ITS CONVERGENCE ANALYSIS}

First we treat the case where there is no observation noise and no frequency mismatch. The input signals are assumed to be pure complex sinusoids where the frequencies $\omega_{1}, \omega_{2}, \ldots, \omega_{p}$ are known and distinct. The desired signal $d(n)$ is modeled as

$$
d(n)=\sum_{i=1}^{p} w_{o i}^{*} e^{j \omega_{i} n} .
$$

where $(\cdot)^{*}$ denotes the complex conjugate. The input vector to the adaptive filter is

$$
\boldsymbol{x}(n)=\left(e^{j \omega_{1} n} e^{j \omega_{2} n} \cdots e^{j \omega_{p} n}\right)^{T}
$$

where $(\cdot)^{T}$ denotes the transpose. The tap weight vector of the adaptive filter is

$$
\boldsymbol{w}(n)=\left[w_{1}(n) w_{2}(n) \cdots w_{p}(n)\right]^{T} .
$$

The output of the adaptive filter is

$$
y(n)=\boldsymbol{w}^{H}(n) \boldsymbol{x}(n)
$$

where $(\cdot)^{H}$ denotes the Hermitian transpose. The error signal is

$$
e(n)=d(n)-y(n) .
$$

The complex LMS algorithm with different step size for each tone is

$$
\boldsymbol{w}(n+1)=\boldsymbol{w}(n)+\Lambda_{\mu} \boldsymbol{x}(n) e^{*}(n)
$$

where $\Lambda_{\mu}=\operatorname{diag}\left[\mu_{1}, \mu_{2}, \ldots, \mu_{p}\right]$ and $\mu_{i}$ is the positive step gain. When real-valued systems are treated, we set a pair of frequency $\omega_{i}$ and $-\omega_{i}$ with the same step size and complex conjugate pair of initial weights. The desired convergent point of $\boldsymbol{w}(n)$ is

$$
\boldsymbol{w}_{o}=\left(w_{o 1} w_{o 2} \cdots w_{o p}\right)^{T}
$$

and the tap error vector is defined by

$$
\boldsymbol{v}(n)=\boldsymbol{w}(n)-\boldsymbol{w}_{o} .
$$

From (1), (3) and (4) the $i$-th element of $\boldsymbol{v}(n)$ is written as

$$
v_{i}(n+1)=v_{i}(n)-\mu_{i} e^{j \omega_{i} n} \sum_{k=1}^{p} e^{-j \omega_{k} n} v_{k}(n) .
$$

Defining $z_{i}(n) \equiv e^{-j \omega_{i} n} v_{i}(n)$, we have

$$
z_{i}(n+1)=e^{-j \omega_{i}} z_{i}(n)-\mu_{i} e^{-j \omega_{i}} \sum_{k=1}^{p} z_{k}(n) .
$$


Thus a time-invariant system is obtained in a much simpler way than in [4]. Defining $\boldsymbol{z}(n)=\left[z_{1}(n) z_{2}(n) \cdots z_{p}(n)\right]^{T},(5)$ is written as

$$
\boldsymbol{z}(n+1)=\Lambda_{\omega}^{*}\left(\boldsymbol{I}-\boldsymbol{\mu} \boldsymbol{\pi}^{H}\right) \boldsymbol{z}(n)
$$

with

$$
\begin{aligned}
\Lambda_{\omega} & =\operatorname{diag}\left[e^{j \omega_{1}}, e^{j \omega_{2}}, \ldots, e^{j \omega_{p}}\right], \\
\boldsymbol{\mu} & =\left(\mu_{1} \mu_{2} \cdots \mu_{p}\right)^{T}, \boldsymbol{\pi}=\left(\begin{array}{llll}
1 & 1 & \cdots & 1
\end{array}\right)^{T} .
\end{aligned}
$$

A necessary and sufficient condition for convergence of $\boldsymbol{z}(n)$ to $\mathbf{0}$ is obtained as follows. Since $\left|\operatorname{det} \Lambda_{\omega}^{*}\left(\boldsymbol{I}-\boldsymbol{\mu} \boldsymbol{\pi}^{H}\right)\right|=\mid 1-$ $\sum_{i=1}^{p} \mu_{i} \mid$, obviously the condition

$$
2-\sum_{i=1}^{p} \mu_{i}>0
$$

is necessary. The sufficiency is proved by using the wellknown fact in linear system theory. That is, let a $p$ dimensional system be

$$
\boldsymbol{z}(n+1)=\boldsymbol{F}^{H} \boldsymbol{z}(n)
$$

then this system is stable if a Lyapunov function $V(n)$ exists such that

$$
\begin{aligned}
V(n) & =\boldsymbol{z}^{H}(n) \boldsymbol{P} \boldsymbol{z}(n) \\
V(n+1)-V(n) & =\boldsymbol{z}^{H}(n)\left(\boldsymbol{F} \boldsymbol{P} \boldsymbol{F}^{H}-\boldsymbol{P}\right) \boldsymbol{z}(n) \\
& =-\boldsymbol{z}^{H}(n) \boldsymbol{L} \boldsymbol{L}^{H} \boldsymbol{z}(n) \leq 0
\end{aligned}
$$

where $\boldsymbol{P}$ satisfies the Lyapunov equation

$$
\boldsymbol{F} \boldsymbol{P} \boldsymbol{F}^{H}-\boldsymbol{P}=-\boldsymbol{L} \boldsymbol{L}^{H}
$$

and $\boldsymbol{P}$ is a positive definite matrix. If $\boldsymbol{L} \boldsymbol{L}^{H}$ is nonsingular, $\boldsymbol{F}$ is a stable matrix. If $\boldsymbol{L} \boldsymbol{L}^{H}$ is singular, but the following reachability matrix

$$
\boldsymbol{W}=\left[\boldsymbol{L} \boldsymbol{F} \boldsymbol{L} \cdots \boldsymbol{F}^{p-1} \boldsymbol{L}\right]
$$

is of full rank, that is, $\operatorname{rank} \boldsymbol{W}=p$, then $\boldsymbol{F}$ is stable. To check this, PBH (Popov-Belevich-Hautus) test [6, Chapter 16] is used, that is,

$$
\begin{aligned}
& \operatorname{rank} \boldsymbol{W}=p \Leftrightarrow \text { for all } \lambda \in C \\
& \qquad \boldsymbol{z}^{H}\left[\begin{array}{ll}
\boldsymbol{F}-\lambda \boldsymbol{I} & \boldsymbol{L}
\end{array}\right]=\mathbf{0}^{H} \text { means } \boldsymbol{z}=\mathbf{0} .
\end{aligned}
$$

In our case for the Lyapunov equation (9) we readily find the following correspondence as

$$
\begin{aligned}
\boldsymbol{F} & \rightarrow\left(\boldsymbol{I}-\boldsymbol{\pi} \boldsymbol{\mu}^{H}\right) \Lambda_{\omega}, \\
\boldsymbol{P} & \rightarrow \operatorname{diag}\left[\frac{1}{\mu_{1}}, \frac{1}{\mu_{2}}, \ldots, \frac{1}{\mu_{p}}\right], \\
\boldsymbol{L} & \rightarrow \sqrt{2-\sum_{i=1}^{p} \mu_{i}} \boldsymbol{\pi}, \quad\left(2>\sum_{i=1}^{p} \mu_{i}\right) .
\end{aligned}
$$

Then the PBH test becomes

$$
\Lambda_{\omega}^{*}\left(\boldsymbol{I}-\boldsymbol{\mu} \boldsymbol{\pi}^{H}\right) \boldsymbol{z}=\lambda^{*} \boldsymbol{z}, \boldsymbol{\pi}^{H} \boldsymbol{z}=0 .
$$

So $\Lambda_{\omega}^{*} z=\lambda^{*} z$. Hence, $e^{-j \omega_{i}} z_{i}=\lambda^{*} z_{i}(i=1, \ldots, p)$. If $\lambda^{*} \neq$ $e^{-j \omega_{1}}, \ldots, e^{-j \omega_{p}}, z_{i}=0$ and $z=0$. If $\lambda^{*}=e^{-j \omega_{k}}$, then $z_{i}=$ $0(i \neq k)$ but $\boldsymbol{\pi}^{H} \boldsymbol{z}=z_{k}=0$. This means $\boldsymbol{z}=\mathbf{0}$. Thus, (8) is also sufficient. The sufficiency can be seen by the general method in [2, Chapter 6]. But here the necessity has been also shown. Also, the time-invariant system (6) is needed for later developments.

\section{THE TRANSIENT ANALYSIS}

Next as a measure of transient behavior, the sum of the squared magnitude of the error signals is considered. Since the error signal is written as $e^{*}(n)=-\boldsymbol{\pi}^{H} \boldsymbol{z}(n)$, from (6) we have

$$
\begin{aligned}
\sum_{n=0}^{\infty}|e(n)|^{2} & =\sum_{n=0}^{\infty}\left|\boldsymbol{\pi}^{H} \boldsymbol{z}(n)\right|^{2} \\
& =\boldsymbol{z}^{H}(0) \sum_{n=0}^{\infty} \boldsymbol{F}^{n} \boldsymbol{\pi} \boldsymbol{\pi}^{H}\left(\boldsymbol{F}^{H}\right)^{n} \boldsymbol{z}(0) \\
& =\boldsymbol{z}^{H}(0) \boldsymbol{Q} \boldsymbol{z}(0)
\end{aligned}
$$

where $\boldsymbol{Q}$ satisfies the following Lyapunov equation

$$
\boldsymbol{Q}=\boldsymbol{F} \boldsymbol{Q} \boldsymbol{F}^{H}+\boldsymbol{\pi} \boldsymbol{\pi}^{H}
$$

with $\boldsymbol{F}^{H}=\Lambda_{\omega}^{*}\left(\boldsymbol{I}-\boldsymbol{\mu} \boldsymbol{\pi}^{H}\right)$. Since $\boldsymbol{F}$ is stable, (12) has a unique solution. That is,

$$
\boldsymbol{Q}=\frac{1}{2-\sum_{i=1}^{p} \mu_{i}} \operatorname{diag}\left[\frac{1}{\mu_{1}}, \frac{1}{\mu_{2}}, \ldots, \frac{1}{\mu_{p}}\right] .
$$

Hence we have

$$
\sum_{n=0}^{\infty}|e(n)|^{2}=\frac{\sum_{i=1}^{p}\left(\left|z_{i}(0)\right|^{2} / \mu_{i}\right)}{2-\sum_{i=1}^{p} \mu_{i}} \equiv f(\boldsymbol{\mu}) .
$$

By minimizing this quantity, an optimal choice of $\boldsymbol{\mu}$ is obtained. From $\partial f(\boldsymbol{\mu}) / \partial \mu_{i}=0$, we have

$$
\frac{\mu_{i}^{2}}{\alpha_{i}}=\frac{2-\sum_{k=1}^{p} \mu_{k}}{\sum_{k=1}^{p}\left(\alpha_{k} / \mu_{k}\right)} \equiv c
$$

where $\alpha_{i}=\left|z_{i}(0)\right|^{2}$. Hence, $\mu_{i}=\sqrt{c \alpha_{i}}$ and

$$
\frac{2-\sum_{k=1}^{p} \sqrt{\alpha_{k}} \sqrt{c}}{(1 / \sqrt{c}) \sum_{k=1}^{p} \sqrt{\alpha_{k}}}=c .
$$

So $\sqrt{c}=\left(\sum_{k=1}^{p} \sqrt{\alpha_{k}}\right)^{-1}$ and the optimal step size is given by

$$
\mu_{i}^{\mathrm{opt}}=\frac{\sqrt{\alpha_{i}}}{\sum_{k=1}^{p} \sqrt{\alpha_{k}}}(i=1, \ldots, p) .
$$

Since (14) contains the unknown true weight vector $\boldsymbol{w}_{o}$, we can not use this. But at least the optimal selection must satisfy

$$
\sum_{i=1}^{p} \mu_{i}^{\mathrm{opt}}=1
$$

\section{THE EFFECT OF OBSERVATION NOISE}

In the case that observation noise is present, the exact expression of the excess mean square error (EMSE) is derived. Instead of (1), the desired signal is written as

$$
d(n)=\sum_{i=1}^{p} w_{o i}^{*} e^{j \omega_{i} n}+\varepsilon^{*}(n)
$$

where $\varepsilon(n)$ is white noise with mean 0 and the variance $E\left[|\varepsilon(n)|^{2}\right]=\sigma^{2}$. Then, (6) is rewritten as

$$
\boldsymbol{z}(n+1)=\boldsymbol{F}^{H} \boldsymbol{z}(n)+\Lambda_{\omega}^{*} \boldsymbol{\mu} \varepsilon(n) .
$$


Let the covariance matrix of $\boldsymbol{z}(n)$ be $\boldsymbol{Z}(n)$. Then

$$
\boldsymbol{Z}(n+1)=\boldsymbol{F}^{H} \boldsymbol{Z}(n) \boldsymbol{F}+\Lambda_{\omega}^{*} \boldsymbol{\mu} \boldsymbol{\mu}^{H} \Lambda_{\omega} \sigma^{2}
$$

and the limit of $\boldsymbol{Z}(n)$ satisfies the following Lyapunov equation

$$
\boldsymbol{Z}=\boldsymbol{F}^{H} \boldsymbol{Z} \boldsymbol{F}+\Lambda_{\omega}^{*} \boldsymbol{\mu} \boldsymbol{\mu}^{H} \Lambda_{\omega} \sigma^{2}
$$

This equation also has a unique solution which is given by

$$
\boldsymbol{Z}=\frac{\sigma^{2}}{2-\sum_{i=1}^{p} \mu_{i}} \operatorname{diag}\left[\mu_{1}, \mu_{2}, \ldots, \mu_{p}\right]
$$

In this case, the error signal is written as

$$
e^{*}(n)=\varepsilon(n)-\boldsymbol{\pi}^{H} \boldsymbol{z}(n),
$$

so the limit of the excess mean square error (EMSE) is given by

$$
\lim _{n \rightarrow \infty} E\left[|e(n)|^{2}\right]-\sigma^{2}=\boldsymbol{\pi}^{H} \mathbf{Z} \boldsymbol{\pi}
$$

Hence from (19) we have

$$
\mathrm{EMSE}=\frac{\sigma^{2} \sum_{i=1}^{p} \mu_{i}}{2-\sum_{i=1}^{p} \mu_{i}}
$$

\section{THE EFFECT OF FREQUENCY MISMATCH}

In some situations the frequencies in the desired signal are not precisely known. Here we treat such a case where the assumed frequencies in the LMS algorithm in (2) are different from those in the desired signal

$$
d(n)=\sum_{i=1}^{p} w_{o i}^{*} e^{j \omega_{o i} n}+\varepsilon^{*}(n)
$$

where $\Delta \omega_{o i}=\omega_{o i}-\omega_{i}(i=1, \ldots, p)$ is the frequency mismatch. From (2), (3) and (21) as in Section 2, the $i$-th element of $\boldsymbol{w}(n)$ is written as

$$
\begin{gathered}
w_{i}(n+1)=w_{i}(n)+\mu_{i} e^{j \omega_{i} n} \varepsilon(n) \\
+\mu_{i} e^{j \omega_{i} n} \sum_{k=1}^{p}\left(e^{-j \omega_{o k} n} w_{o k}-e^{-j \omega_{k} n} w_{k}(n)\right) .
\end{gathered}
$$

Defining the vector $\tilde{z}(n)=\left[\begin{array}{llll}\tilde{z}_{1}(n) & \cdots & \tilde{z}_{p}(n)\end{array}\right]^{T}$ with $\tilde{z}_{i}(n) \equiv$ $e^{-j \omega_{i} n} w_{i}(n)$, we have the following time-invariant system

$$
\tilde{\boldsymbol{z}}(n+1)=\boldsymbol{F}^{H} \tilde{\boldsymbol{z}}(n)+\Lambda_{\omega}^{*} \boldsymbol{\mu} \varepsilon(n)+\Lambda_{\omega}^{*} \boldsymbol{\mu} \boldsymbol{\pi}^{H}\left(\Lambda_{o \omega}^{*}\right)^{n} \boldsymbol{w}_{o}
$$

with

$$
\begin{aligned}
\boldsymbol{F}^{H} & =\Lambda_{\omega}^{*}\left(\boldsymbol{I}-\boldsymbol{\mu} \boldsymbol{\pi}^{H}\right), \\
\Lambda_{o \omega} & =\operatorname{diag}\left[e^{j \omega_{o 1}}, e^{j \omega_{o 2}}, \ldots, e^{j \omega_{o p}}\right] .
\end{aligned}
$$

So,

$$
\begin{aligned}
\tilde{z}(n)= & \sum_{i=0}^{n-1}\left(\boldsymbol{F}^{H}\right)^{n-1-i} \Lambda_{\omega}^{*} \boldsymbol{\mu} \varepsilon(i) \\
+ & \sum_{i=0}^{n-1}\left(\boldsymbol{F}^{H}\right)^{n-1-i} \Lambda_{\omega}^{*} \boldsymbol{\mu} \boldsymbol{\pi}^{H}\left(\Lambda_{o \omega}^{*}\right)^{i} \boldsymbol{w}_{o}+\left(\boldsymbol{F}^{H}\right)^{n} \tilde{\boldsymbol{z}}(0) .
\end{aligned}
$$

The error signal is now written as

$$
\begin{aligned}
& e^{*}(n)=\boldsymbol{\pi}^{H}\left(\Lambda_{o \omega}^{*}\right)^{n} \boldsymbol{w}_{o}-\boldsymbol{\pi}^{H} \sum_{i=0}^{n-1}\left(\boldsymbol{F}^{H}\right)^{n-1-i} \Lambda_{\omega}^{*} \boldsymbol{\mu} \varepsilon(i) \\
& -\boldsymbol{\pi}^{H} \sum_{i=0}^{n-1}\left(\boldsymbol{F}^{H}\right)^{n-1-i} \Lambda_{\omega}^{*} \boldsymbol{\mu} \boldsymbol{\pi}^{H}\left(\Lambda_{o \omega}^{*}\right)^{i} \boldsymbol{w}_{o} \\
& -\boldsymbol{\pi}^{H}\left(\boldsymbol{F}^{H}\right)^{n} \tilde{\boldsymbol{z}}(0)+\varepsilon(n)
\end{aligned}
$$

The matrix sum in the third term of the right hand side of (23) which is denoted by $\boldsymbol{\Phi}$ can be rewritten as

$$
\begin{aligned}
\boldsymbol{\Phi} & =\sum_{i=0}^{n-1}\left(\boldsymbol{F}^{H}\right)^{n-1-i} \Lambda_{\omega}^{*} \boldsymbol{\mu} \boldsymbol{\pi}^{H}\left(\Lambda_{o \omega}^{*}\right)^{i} \\
& =\left[\sum_{i=0}^{n-1}\left(\boldsymbol{F}^{H}\right)^{i} \Lambda_{\omega}^{*} \boldsymbol{\mu} \boldsymbol{\pi}^{H}\left(\Lambda_{o \omega}\right)^{i}\right]\left(\Lambda_{o \omega}^{*}\right)^{n-1} .
\end{aligned}
$$

To calculate this sum, we use the following identity. That is, let

$$
\boldsymbol{Y}(n)=\sum_{i=0}^{n} \boldsymbol{A}^{i} \boldsymbol{C} \boldsymbol{B}^{i}, \quad \boldsymbol{Y}(0)=\boldsymbol{C},
$$

then $\boldsymbol{Y}(n)=\boldsymbol{D}+\boldsymbol{A}^{n}(\boldsymbol{C}-\boldsymbol{D}) \boldsymbol{B}^{n}$ where $\boldsymbol{D}$ satisfies

$$
D=C+A D B
$$

The solution $\boldsymbol{D}$ of (24) exists if the product of any pair of the eigenvalues of $\boldsymbol{A}$ and $\boldsymbol{B}$ is not equal to 1. Corresponding to (24) we have

$$
\boldsymbol{D}=\Lambda_{\omega}^{*} \boldsymbol{\mu} \boldsymbol{\pi}^{H}+\boldsymbol{F}^{H} \boldsymbol{D} \Lambda_{o \omega} .
$$

The matrix equation (25) can be written as

$$
\left(-\boldsymbol{I}+\Lambda_{o \omega}^{T} \otimes \boldsymbol{F}^{H}\right) \operatorname{vec} \boldsymbol{D}=-\operatorname{vec}\left(\Lambda_{\omega}^{*} \boldsymbol{\mu} \boldsymbol{\pi}^{H}\right)
$$

where $\otimes$ denotes the Kronecker product and vec denotes the operation that transforms a matrix into a vector by stacking the columns of the matrix. Since the absolute value of any eigenvalue of $\boldsymbol{F}^{H}$ is less than 1 and that of $\Lambda_{o \omega}$ is 1 , a unique solution exists. Defining $\boldsymbol{D}=\left[\begin{array}{llll}\boldsymbol{d}_{1} & \boldsymbol{d}_{2} & \cdots & \boldsymbol{d}_{p}\end{array}\right]$ and using the identity $\operatorname{vec}\left(\boldsymbol{a} \boldsymbol{b}^{T}\right)=\boldsymbol{b} \otimes \boldsymbol{a},(26)$ can be rewritten as

$$
\begin{aligned}
& {\left[\begin{array}{ccc}
-\boldsymbol{I}+e^{j \omega_{o 1}} \boldsymbol{F}^{H} & & 0 \\
& \ddots & \\
\mathbf{0} & & -\boldsymbol{I}+e^{j \omega_{o p}} \boldsymbol{F}^{H}
\end{array}\right]\left[\begin{array}{c}
\boldsymbol{d}_{1} \\
\vdots \\
\boldsymbol{d}_{p}
\end{array}\right] } \\
=-\left[\Lambda_{\omega}^{*} \boldsymbol{\mu} \cdots \Lambda_{\omega}^{*} \boldsymbol{\mu}\right]^{T} . &
\end{aligned}
$$

Hence we have

$$
\left(-\boldsymbol{I}+e^{j \omega_{o i}} \boldsymbol{F}^{H}\right) \boldsymbol{d}_{i}=-\Lambda_{\omega}^{*} \boldsymbol{\mu}
$$

To solve (27) we use the matrix inversion lemma

$$
(\boldsymbol{A}+\boldsymbol{B C})^{-1}=\boldsymbol{A}^{-1}-\boldsymbol{A}^{-1} \boldsymbol{B}\left(\boldsymbol{I}+\boldsymbol{C} \boldsymbol{A}^{-1} \boldsymbol{B}\right)^{-1} \boldsymbol{C A} \boldsymbol{A}^{-1} .
$$

The correspondence is $\boldsymbol{A} \rightarrow-\boldsymbol{I}+e^{j \omega_{o i}} \Lambda_{\omega}^{*}, \quad \boldsymbol{B} \rightarrow$ $-e^{j \omega_{o i}} \Lambda_{\omega}^{*} \boldsymbol{\mu}, \boldsymbol{C} \rightarrow \boldsymbol{\pi}^{H}$. In (23) we need the quantity $\boldsymbol{\pi}^{H} \boldsymbol{d}_{i}$. After some calculations we have

$$
\boldsymbol{\pi}^{H} \boldsymbol{d}_{i}=-e^{-j \omega_{o i}}\left(1+\frac{1}{\gamma_{i}-1}\right)
$$

where

$$
\gamma_{i}=\sum_{k=1}^{p} \frac{\mu_{k} e^{j\left(\omega_{o i}-\omega_{k}\right)}}{-1+e^{j\left(\omega_{o i}-\omega_{k}\right)}} .
$$

Using the solution $\boldsymbol{D}$ of (25) we have

$$
\boldsymbol{\Phi}=\left[\boldsymbol{D}+\left(\boldsymbol{F}^{H}\right)^{n}\left(\Lambda_{\omega}^{*} \boldsymbol{\mu} \boldsymbol{\pi}^{H}-\boldsymbol{D}\right)\left(\Lambda_{o \omega}\right)^{n}\right]\left(\Lambda_{o \omega}^{*}\right)^{n-1} .
$$

Hence, in the steady-state, that is, $n \rightarrow \infty$, since $\left(\boldsymbol{F}^{H}\right)^{n} \rightarrow$ $\mathbf{0}$, the deterministic component of $e^{*}(n)$ in (23) is given by $\boldsymbol{\pi}^{H}\left[\Lambda_{o \omega}^{*}-\boldsymbol{D}\right]\left(\Lambda_{o \omega}^{*}\right)^{n-1} \boldsymbol{w}_{o}$. Thus, using (28) this component is finally written as

$$
-\sum_{i=1}^{p} \frac{1}{\gamma_{i}-1} w_{o i} e^{-j \omega_{o i} n}
$$




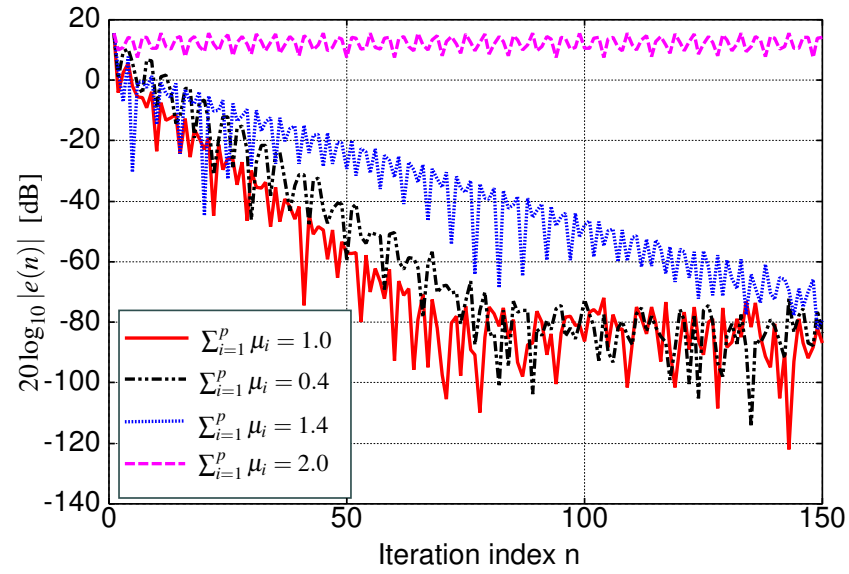

Figure 1: Learning Curves in $\mathrm{dB}$ for $\sum_{i=1}^{p} \mu_{i}=1.0,0.4,1.4$ and 2.0.

This term is generated due to the frequency mismatch. This is the exact steady-state expression for the deterministic component in $e(n)$. We note that if there is no frequency mismatch, that is, $\omega_{o i}=\omega_{i}$, then $\gamma_{i}=\infty$ and (30) is zero as it should. The increase in the time averaged squared magnitude of the error signals due to the frequency mismatch is given by

$$
\operatorname{MISMATCH}=\sum_{i=1}^{p} \frac{1}{\left|\gamma_{i}-1\right|^{2}}\left|w_{o i}\right|^{2} .
$$

If the frequency mismatch $\Delta \omega_{i}$ is sufficiently small, the $i$-th term in (29) is dominant and (31) is approximated as

$$
\begin{aligned}
\operatorname{MISMATCH} & \simeq \sum_{i=1}^{p} \frac{2\left(1-\cos \Delta \omega_{i}\right)}{\mu_{i}^{2}}\left|w_{o i}\right|^{2} \\
& \simeq \sum_{i=1}^{p} \frac{\left(\Delta \omega_{i}\right)^{2}}{\mu_{i}^{2}}\left|w_{o i}\right|^{2} .
\end{aligned}
$$

\section{SIMULATION RESULTS}

To examine the validity of the above theoretical developments, some simulations have been performed. We assume that the reference signal vector is given by $\boldsymbol{x}(n)=\left(e^{j \pi n / 4} e^{-j \pi n / 4} e^{j \pi n / 2} e^{-j \pi n / 2}\right)^{T}$ with $p=4$ sources and the optimal weight $\boldsymbol{w}_{o}=(1+j 1-j-j / 4+j / 4)^{H}$. Hereafter, we put that $\mu_{1}=\mu_{2}$ and $\mu_{3}=\mu_{4}$ and that various combinations are considered as $\left(\mu_{1} \mu_{3}\right)=(0.10 .1),(0.150 .1),(0.30 .2),(0.40 .3)$ and $(0.60 .4)$ with $\sum_{i=1}^{p} \mu_{i}=0.4,0.5,1.0,1.4$ and 2.0 , respectively. As for observation noise $\varepsilon(n)$, white noise is added to $d(n)$ with mean 0 and the variance $\sigma^{2}=10^{-8}$. Initial weights are set to $w_{i}(0)=1(i=1,2,3,4)$. Furthermore, in Tables 1-2, we show the time averages of the squared magnitude of the error signals from the iteration index 1000 to 9999 . First, to confirm the validity of convergence condition in (8) and optimality condition in (15), simulations are carried out by using various values of $\boldsymbol{\mu}$. Fig. 1 shows the convergence characteristics with $\sum_{i=1}^{p} \mu_{i}=0.4,1.0,1.4$ and 2.0 , respectively. From Fig. 1 we see that the combination satisfying (15) gives the best result and the stability limit in (8) is correct. Next, to clarify the validity of EMSE in (20), the simulation results are shown in Table. 1. The theoretical values of EMSE are good agreement with the experimental
Table 1: Experimental and theoretical values of the limit of the excess mean square error (EMSE).

\begin{tabular}{|l||c|c|}
\hline & $\sum_{i=1}^{p} \mu_{i}=0.5$ & $\sum_{i=1}^{p} \mu_{i}=1.0$ \\
\hline experimental value & $3.39 \times 10^{-9}$ & $1.05 \times 10^{-8}$ \\
\hline value in $(20)$ & $3.33 \times 10^{-9}$ & $1.00 \times 10^{-8}$ \\
\hline
\end{tabular}

Table 2: Experimental and theoretical values of the increase in the time averaged squared magnitude of the error signals due to the frequency mismatch.

\begin{tabular}{|l||c|c|}
\hline & $a=1^{\dagger}$ & $a=0.1$ \\
\hline experimental value & $6.38 \times 10^{-2}$ & $7.06 \times 10^{-4}$ \\
\hline value in (31) & $6.38 \times 10^{-2}$ & $7.06 \times 10^{-4}$ \\
\hline value in (32) & $7.16 \times 10^{-2}$ & $7.16 \times 10^{-4}$ \\
\hline
\end{tabular}

(true signal frequency $^{\dagger}: \omega_{o 1}=\pi / 4+0.01 a \pi, \omega_{o 2}=-\pi / 4-$ $0.01 a \pi, \omega_{o 3}=\pi / 2+0.03 a \pi, \omega_{o 4}=-\pi / 2-0.03 a \pi$. userspecified frequencies: $\quad \omega_{1}=\pi / 4, \omega_{2}=-\pi / 4, \omega_{3}=$ $\pi / 2, \omega_{4}=-\pi / 2$.)

ones. Finally, we compare the experimental and theoretical values in (31) and (32) of the increase in the time averaged squared magnitude of the error signals due to the frequency mismatch. The simulation results are shown in Table. 2 . From Table. 2, since there is no approximation in (31), the result perfectly coincides with the experimental one. But (32) is derived under the assumption that the frequency mismatch $\Delta \omega_{i}$ is sufficiently small, so when the mismatch is large for $a=1$, compared with the case of $a=0.1$, the theoretical value has a relatively large difference with the experimental one.

\section{CONCLUSION}

An exact analysis of the LMS algorithm with tonal reference signals has been presented using the tools of linear system theory. A necessary and sufficient condition is derived and the expressions for excess mean square error and effect of frequency mismatch are also obtained. These powerful tools may be applied to many other problems in adaptive signal processing.

\section{REFERENCES}

[1] B. Widrow and S. D. Stearns, Adaptive Signal Processing, Prentice Hall, 1985.

[2] V. Solo and X. Kong, Adaptive Signal Processing Algorithms - Stability and Performance, Prentice Hall, 1995.

[3] M. Rupp and A. H. Sayed, "A time-domain feedback analysis of filtered-error adaptive gradient algorithms," IEEE Trans. Signal Processing, vol. 44, no. 6, pp. 14281439, June 1996.

[4] S. Johansson, S. Nordebo, and I. Claesson, "Convergence analysis of a twin-reference complex least-meansquares algorithm," IEEE Trans. Speech Audio Processing, vol. 10, no. 4, pp. 213-221, May 2002.

[5] Y. Xiao, R. Ward, and L. Xu, "A new LMS-based Fourier analyzer in the presence of frequency mismatch," in Proc. ISCAS, Bangkok, Thailand, May 2003, vol. 4, pp. 369-372.

[6] A. H. Sayed, Fundamentals of Adaptive Filtering, Wiley, 2003. 